\title{
Editorial
}

\section{Mathematical Modelling and Algorithms in Finance}

\author{
Wei Chen, ${ }^{1}$ Wei-Guo Zhang, ${ }^{2}$ and Pankaj Gupta ${ }^{3}$ \\ ${ }^{1}$ School of Information, Capital University of Economics and Business, Beijing 100070, China \\ ${ }^{2}$ School of Business Administration, South China University of Technology, Guangzhou 510641, China \\ ${ }^{3}$ Department of Operational Research, University of Delhi, Delhi 110007, India
}

Correspondence should be addressed to Wei Chen; chenwei@cueb.edu.cn

Received 24 June 2014; Accepted 24 June 2014; Published 5 August 2014

Copyright (C) 2014 Wei Chen et al. This is an open access article distributed under the Creative Commons Attribution License, which permits unrestricted use, distribution, and reproduction in any medium, provided the original work is properly cited.

In recent years, more and more financial management problems, such as portfolio selection, asset pricing, risk management, and asset-liability management problems, have been presented in the literature adopting formulation and solution approaches rooted in mathematical theory, as well as optimization methods.

In this special issue, we tried to include the variety of the topics which are currently investigated with respect to mathematical modelling and algorithms to the financial management problems, including portfolio selection, asset pricing and hedging, futures and options, and financial forecasting. Below, a very brief overview of the featured works is given.

Portfolio Selection. Three contributions focus on the topics of portfolio selection. In "Portfolio selection with subsistence consumption constraints and CARA utility," by G. Shim and Y. H. Shin, the authors discuss the optimal consumption and portfolio choice problem with constant absolute risk aversion (CARA) utility and a subsistence consumption constraint. They obtain closed form solution of the proposed model by dynamic programming approach. In the paper entitled " Riskcontrolled multiobjective portfolio selection problem using a principle of compromise," T. Hasuike and H. Katagiri present a robust-based multiobjective portfolio selection model with most probable random distribution derived from current market data and other random distributions of boom and recession. X. Yu et al., in the paper entitled "Optimal portfolio strategy under rolling economic maximum drawdown constraints," deal with the problem of optimal portfolio strategy under rolling economic maximum drawdown constraint.
The authors propose two new strategies called "Rolling Sharpe Ratio Strategy" and "Rolling Economic Drawdown of Risky Asset's Prices Strategy," respectively.

Asset Pricing and Hedging. Two papers published in this special issue are focused on asset pricing and hedging strategy. The paper entitled "Valuing catastrophe bonds involving credit risks," by J. Liu et al. proposes a CAT bonds pricing model with credit risk, in which Jarrow and Turnbull method is used to model the credit risks. Y. Liu et al. investigate the problem of the rollover hedge strategy for the long-term exposure of a well-diversified portfolio in "Hedging long-term exposures of a well-diversified portfolio with short-term stock index futures contracts." The authors propose a rollover hedging model for the well-diversified portfolio and then describe how to solve the proposed model by the equivalent transformation technique and dynamic programming.

Futures and Options. Three papers focus on the topics of futures and options. In "An agent-based computational model for China's stock market and stock index futures market," H.$\mathrm{C}$. $\mathrm{Xu}$ et al. develop an agent-based computational model with spot-futures cross market structure that coincides with the main characteristics of the Chinese stock market and the CSI index futures market. Empirical research indicates that the proposed model can successfully reproduce the Chinese financial markets' features. The paper entitled "Canonical least-squares Monte Carlo valuation of American options: convergence and empirical pricing analysis," by X. Yu and Q. Liu discusses the convergence properties of the canonical least-squares Monte Carlo (CLM) method for American 
options. Based on empirical analysis, the authors show that the estimated price can converge to the true price at a given rate by using CLM method, and this method is superior to the benchmark methods of binominal tree and finite difference with historic volatilities. In the paper entitled "Pricing extendible options using the fast Fourier transform," S. N. I. Ibrahim et al. apply the fast Fourier transform (FFT) approach to derive a closed-form solution for holder-extendible call options. Compared to Monte Carlo simulation, numerical examples demonstrate that the FFT is both computationally more efficient and higher in accuracy.

Financial Forecasting. Five papers deal with financial forecasting problem by different methods. In the paper entitled "Modeling the dynamics of Shanghai interbank offered rate based on single-factor short rate processes," X. Zhang proposes the maximum likelihood methodology to estimate the parameters of five single-factor interest rate models including Merton, GBM, Vasicek, CIR, and MRJD and compares these five different models of short-term interest rate dynamics in order to determine which model best fits the SHIBOR data. In the paper "Forecasting crude oil price with multiscale denoising ensemble model," by X. Li et al., the authors propose a novel crude oil price forecasting methodology WDN-ICA-LSSVR, in which ICA is used to reduce the forecast matrix dimensions and the LSSVR based nonlinear ensemble algorithm is used to ensemble partial information set. L. Wang et al., in the paper "Generating moving average trading rules on the oil futures market with genetic algorithms," apply genetic algorithms to generate moving average trading rules in the oil futures market. The authors compare the proposed trading rules with the buyand-hold strategy and conclude that the proposed trading rules can help trader make profits when there are obvious price fluctuations. In the paper entitled "Financial time series forecasting using directed-weighted chunking SVMs," Y. Cai et al. propose a directed-weighted chunking SVMs algorithm for financial time series forecasting. Results show that the proposed algorithm can improve the computational speed without reducing prediction accuracy. In the paper "Analysis of multiple structural changes in financial contagion based on largest Lyapunov exponents," R. Wang et al. propose a modified multiple structural change model to test structural breaks of a financial system based on largest Lyapunov exponents. Simulation results show that the new model has good capability in both finding the breakpoint and revealing the changes in nonlinear characteristics of the time series.

Investment and Reinsurance. The paper "Optimal investment and reinsurance for insurers with uncertain time-horizon," by A. Gu et al., discusses the investment-reinsurance problems for an insurer with uncertain time-horizon. Assuming that the insurer can invest his/her surplus in a risky asset and purchase proportional reinsurance, two optimization problems are discussed in the cases of the surplus process described by a jump-diffusion model and a diffusion-approximation model. The expression for optimal investment and reinsurance strategy are derived by dynamic programming approach and Feynman-Kac representation.
Net Present Value Problem. The paper entitled "Possibilistic fuzzy net present value model and application," by S. S. Appadoo, provides some moment properties with special type of fuzzy numbers, called $(m, n)$-trapezoidal fuzzy number, and then proposes a fuzzy net present value model, which does not imply rejection of other discounted cash formulations but rather compliments existing fuzzy model formulation.

The papers, published in this special issue, have novelty and contain some interesting, creative, and prominent ideas. We do believe that all the papers published in this special issue will motivate further scientific activities in the field of mathematical modelling and algorithms in finance.

\section{Acknowledgments}

The guest editors wish to express their sincere gratitude to the authors and reviewers who contributed greatly to the success of this special issue. The preparation of this special collection has been partially supported by the Humanity and Social Science Youth foundation of Ministry of Education of China (no. 13YJC630012).

Wei Chen

Wei-Guo Zhang

Pankaj Gupta 


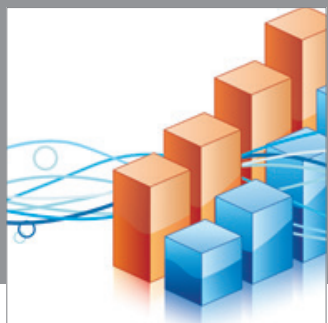

Advances in

Operations Research

mansans

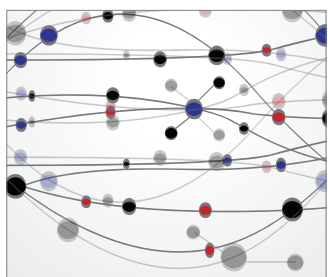

The Scientific World Journal
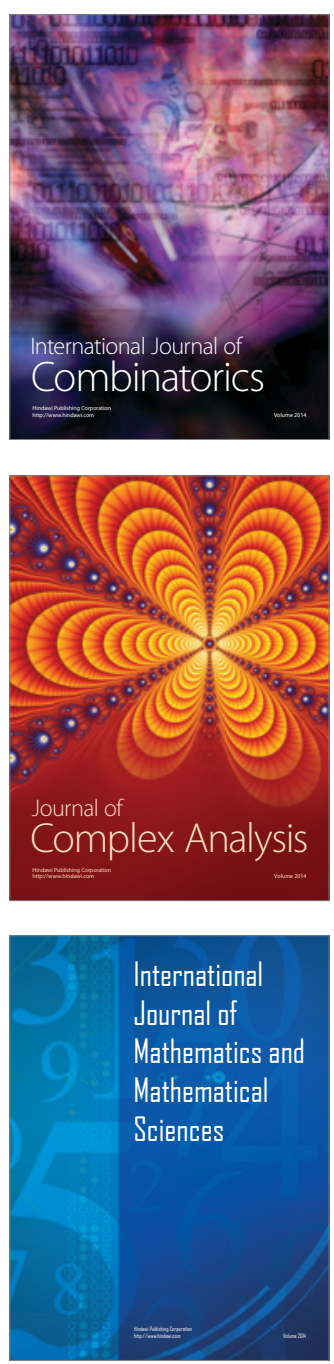
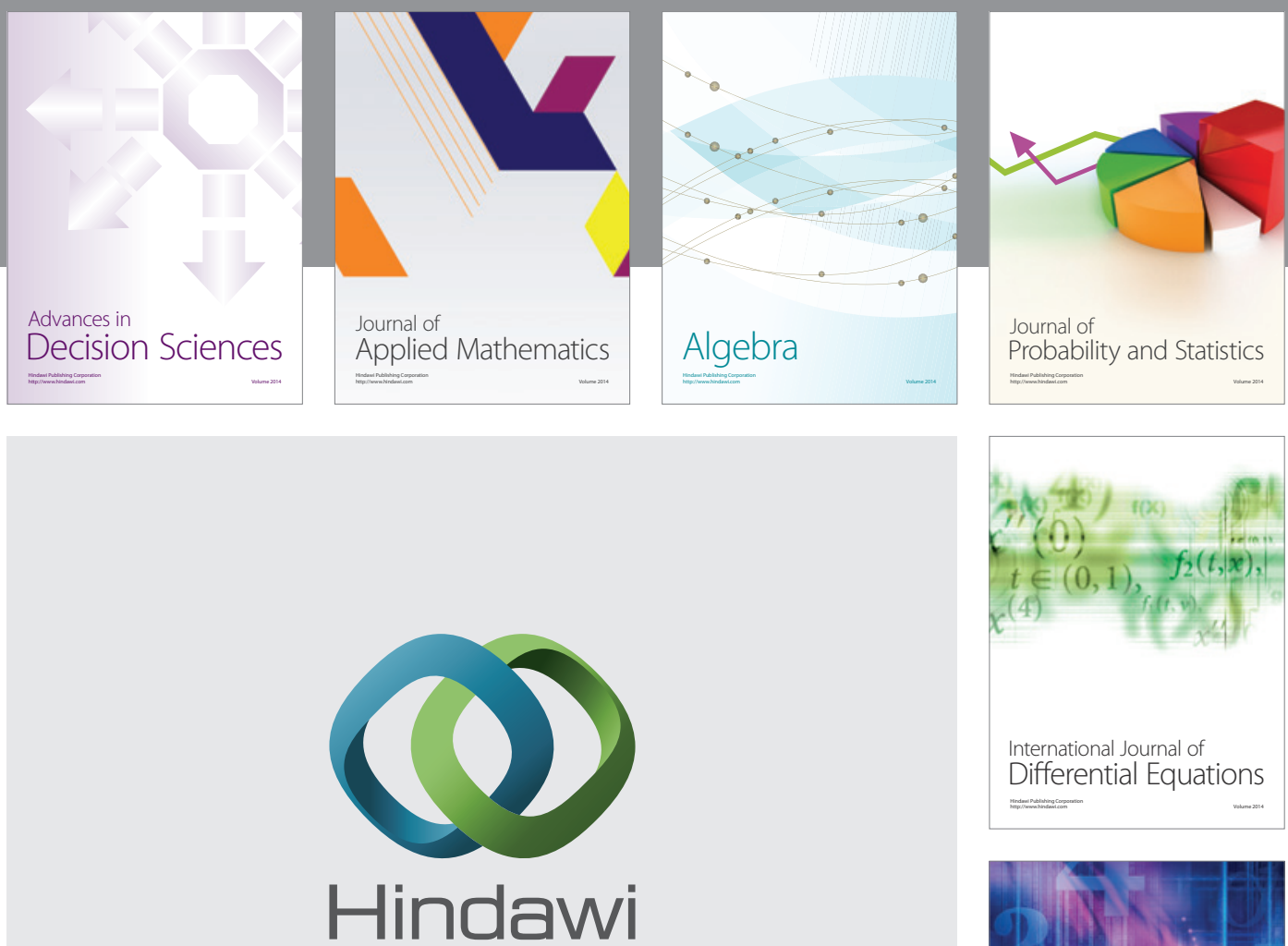

Submit your manuscripts at http://www.hindawi.com
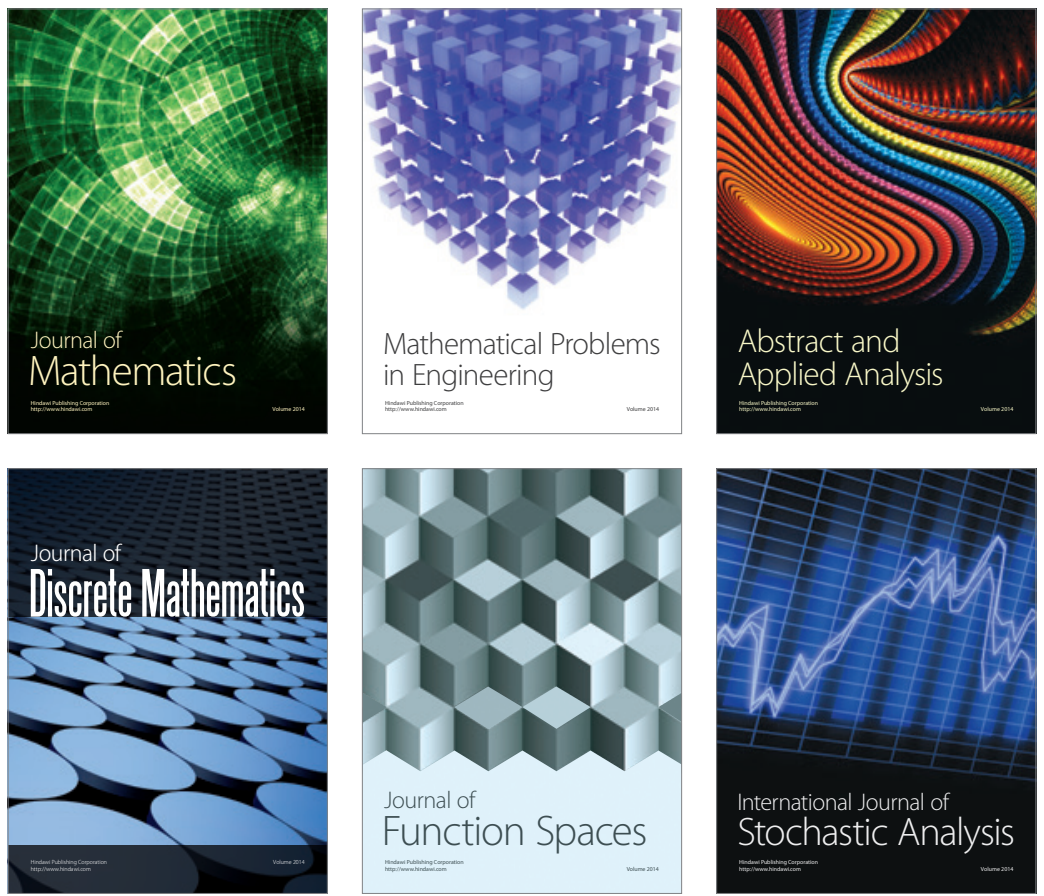

Journal of

Function Spaces

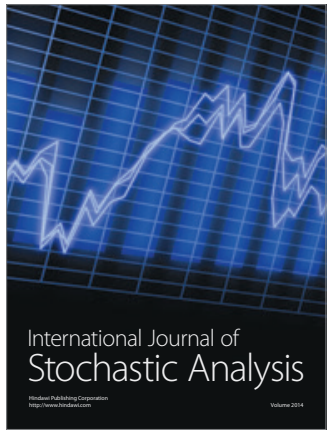

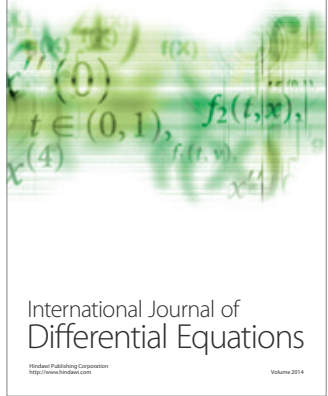
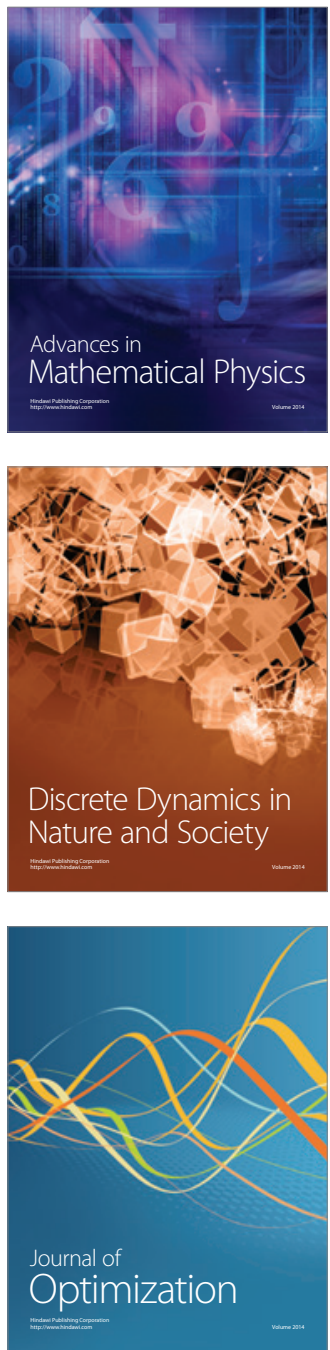